\title{
Review: supplemented enteral nutrition reduces infectious complications and length of hospital stay in patients with critical illness
}

Heys SD, Walker LG, Smith I, et al. Enteral nutritional supplementation with key nutrients in patients with critical illness and cancer. A meta-analysis of randomized controlled clinical trials. Ann Surg 1999 Apr;229:467-77.

QUESTION: Among patients with critical illness, does enteral nutrition supplemented with key nutrients compared with standard enteral nutrition reduce infectious complications, death, and length of hospital stay?

\section{Data sources}

Studies published in peer reviewed journals from 1990 to February 1998 were identified by searching Medline, doing handsearches of journals, and reviewing bibliographies of relevant articles.

\section{Study selection}

Randomised controlled trials were included if they compared the effects of enteral nutrition supplemented with key nutrients with standard enteral nutrition in patients who were critically ill.

\section{Data extraction}

Data were extracted on patient conditions; timing of initiation of feeding; supplemented nutrients, nutritional goals, achieved intake, and weight loss before study; and power calculations. Main outcomes were major infectious complications (wound infection, intra-abdominal abscess, pneumonia, and septicaemia), nosocomial pneumonia alone, death, and length of hospital stay. 2 independent raters assessed the methodological quality (selection, performance, attrition, and detection biases) of each trial.

\section{Main results}

11 trials (1009 patients) were included in the analysis. Enteral supplements included combinations of L-arginine, L-glutamine, branched chain amino acids, essential fatty acids, and ribonucleic acid. Meta-analysis was done on an intention to treat basis, using a fixed effects model. Patients who received supplemented enteral nutrition had a lower rate of infectious complications (table) and a reduced length of hospital stay (8 studies, weighted mean difference in length of stay $2.5 \mathrm{~d}$, $95 \% \mathrm{CI}$ around difference 1.0 to $4.0 \mathrm{~d}$ ) than patients who received standard enteral nutrition. The groups did not differ for rates of nosocomial pneumonia or death. Similar results were found for all outcomes when 6 studies of patients with gastrointestinal cancer $(n=497)$ were analysed separately.

\section{Conclusions}

Among patients with critical illness, enteral nutritional support supplemented with key nutrients reduced

Fax $+44(0) 1224$

Fax $+44(0) 1224$

840973. infectious complications and length of hospital stay when compared with standard enteral nutrition. No differences were found for rates of nosocomial pneumonia or death.

\section{COMMENTARY}

Previous research has shown that certain key nutrients, such as L-arginine, L-glutamine, essential fatty acids, and ribonucleic acid can benefit inflammatory, metabolic, and immune processes, such as wound healing, nitrogen losses after trauma, stimulation of host defenses, and modulation of tumour cell metabolism. ${ }^{12}$ Heys et al completed a metaanalysis of 11 randomised controlled trials designed to evaluate the supplementation of enteral diets with combinations of key nutrients. Rather than examining the effect of the supplemented diet on immunological functions, they focused on clinical outcomes, including infectious complications, length of hospital stay, and death. The strengths of this review include the independent abstraction of data by 2 reviewers, and validity assessment of each trial. They evaluated 7 of the trials as having a low risk of bias, 4 as having a moderate risk of bias, and, fortunately, none had a high risk of bias. The review is weakened, however, by the limited search strategy used to identify studies (ie, only 1 database, Medline, was searched for articles published in the previous 8 y).

The trials included in the analysis were done under slightly different conditions, and nutritional supplements had different contents and were given at different time intervals. It is also not clear whether patient malnutrition was a significant factor in the overall success of the nutritional supplementation.

This review serves as an important consolidation of the evidence to date on enteral nutritional supplementation for critical care nurses. Based on their review, the authors conclude that although supplemented nutritional support may reduce infectious complications and reduce length of hospital stay in patients with critical illness and gastrointestinal cancer, further studies are needed, with particular attention to specific patient groups, defined nutrients in the supplemental formulas, and more precise outcome measures.

Theresa Posani, RN, MS, CS, CNA, CCRN Critical Care Clinical Nurse Specialist Presbyterian Hospital of Dallas Dallas, Texas, USA

1 Heys SD, Gough DB, Khan L, et al. Nutritional pharmacology and malignant disease: a therapeutic modality in patients with cancer. Br J Surg 1996;83:608-19.

2 Heys SD, Park KG, Garlick PJ, et al. Nutrition and malignant disease: implications for surgical practice. $\mathrm{Br} J$ Surg 1992;79:614-23.
Infectious

$\begin{array}{lllll}\text { complications } & 18.4 \% & 28.6 \% & 36 \%(16 \text { to } 51) & 10(7 \text { to } 23)\end{array}$

*Abbreviations defined in glossary; RRR, $\mathrm{Cl}$, and NNT calculated from data in article.

(7)

